\title{
Using the TRS-80 cassette tape recorder as a voice key
}

\author{
THOMAS BIDDLE PERERA \\ Montclair State College, Upper Montclair, New Jersey 07043
}

\begin{abstract}
A simple BASIC language program allows the standard TRS-80 tape recorder to function as a voice key to control the timing or running of an experiment.
\end{abstract}

Voice keys typically cost over $\$ 100$ and require at least some interfacing circuitry to connect to laboratory computers. The standard TRS- 80 cassette recorder can be set up so that any sound picked up by the sensitive built-in microphone will be amplified and sent out to the EAR jack. This amplified voltage may be continually sampled under BASIC program control so that a sound acts as an input during a program.

\section{DESCRIPTION OF HARDWARE}

To set up the recorder to act as a voice-key microphone, the user should unplug the gray plug from the AUX jack of the CTR-80 recorder and leave the black plug in the EAR jack.

Pressing the EJECT key opens the lid and allows you to reach in and overcome the RECORD PROTECT switch. In the left rear corner of the compartment that normally holds the cassette is a small metal bar. The bar is pushed toward the loudspeaker or back of the cassette recorder. While the bar is held back, the PLAY and (red) RECORD keys are depressed simultaneously to lock them. The recorder is now in the record mode without the tape cassette in place. The tape recorder cover is closed, and the recorder is ready for use as a voice key.

\section{DESCRIPTION OF SOFTWARE}

The command A=INP(255) samples the tape recorder's output voltage. If the voltage is less than a critical value, then $A$ is equal to 127 . If the critical value is exceeded, then the value of $A$ is 255 . Low-intensity voices or sounds do not cause the voltage to exceed the critical value. The position of the cassette recorder may be used to adjust sensitivity.

Before the command $\mathrm{A}=\mathrm{INP}(255)$ is used, the cassette recorder must be activated by the command OUT 255,4 . This command must be repeated to reset the INP(255) command after each activation or triggering of the voice key.

\section{Sample Programs}

Program 1. The simplest program to demonstrate the operation of the voice key is the following.

\section{OUT 255,4:PRINT INP(255);:GOTO 100}

This program resets the cassette recorder, prints the value of INP(255), and repeats itself. If you speak near the cassette recorder, the string of $127 \mathrm{~s}$ on the video screen changes to $255 \mathrm{~s}$ as the voice key triggers.

Program 2. The second program continually tests for a volce or a sound, and when one of these is present, it prints a string of "I HEARD IT"s on the screen.

100 OUT 255,4:IF INP(255)> 127 THEN 200 ELSE 100 200 PRINT "I HEARD IT",:GOTO 100

Line 100 resets the input and tests for a number greater than 127 . If it is present, the program goes to Line 200 , prints "I HEARD IT," and restarts. If it is not present, the program simply restarts at Line 100 .

Program 3. The third program measures the elapsed time between pressing the " 1 " key on the keyboard of the computer and the triggering of the voice key. It then prints the latency in seconds on the videoscreen.

$10 \mathrm{~T}=1$ :OUT 255,4

20 IF INKEY $\$=" 1$ " THEN 100 ELSE 10

100 FOR $\mathrm{T}=1$ to 10000

110 IF INP(255) $>127$ THEN 299

120 NEXT T

200 PRINT"SECONDS FROM 1 KEY TO VOICE ="; T/120

This program is started by typing RUN and then pressing the " 1 " key on the keyboard. Any sound or voice causes the latency to be displayed. The user repeats this procedure to run again.

Line 10 initializes the timing counter variable, $T$. This is a critical action that overcomes the initial long delay when a variable is first used and places the variable " $T$ " at the most accessible position at the top of the 
push-down stack. Line 10 also initializes and resets the cassette recorder audio line.

Line 20 tests to determine whether key " 1 " has been depressed. If so, it directs the program to Line 100. Otherwise, it restarts the program.

Line 100 starts the latency counter variable $T$ counting from 1 to 10,000 . Line 110 tests to determine whether a voice has triggered the input line and caused $\operatorname{INP}(255)$ to shift from 127 to 255 . If so, it directs the program to Line 200 . Otherwise, the program goes to Line 120, which directs it to Line 100 to increment the value of $T$ again.

Line 200 prints the label and then prints the current value of $\mathrm{T}$ divided by 120 (a constant used to convert $\mathrm{T}$ to seconds).

The preceding comments should allow anyone familiar with BASIC programming to use this simple voice key. 